To cite this article:

Sels, L. , Schat, E.*, Verhofstadt, L., \& Ceulemans, E. (in press). Introducing change point detection analysis in relationship research: An investigation of couples' emotion dynamics. Journal of Social and Personal Relationships. 
Running Head: CHANGE POINT DETECTION IN INTERPERSONAL DYNAMICS

Introducing Change Point Detection Analysis in Relationship Research: an Investigation of Couples' Emotion Dynamics 


\begin{abstract}
Many relationship theories assume some form of interdependence between relationship partners. Partners are thought to continuously influence each other, and to be influenced by each other over time. These influences are not expected to be constant, but dynamic (sometimes partners influence each other more, and sometimes they do not influence each other). To investigate such changes in interpersonal dynamics, we showcase the value of using a change point detection approach, which can be used to monitor virtually any preferred quantification of interpersonal dynamics across time. Concretely, we introduce the KCP-RS method, which scans times series for changes in user-specified statistics, in interpersonal emotion dynamic research. We used KCP-RS to investigate changes in 96 couples' emotional experiences during two 10-minute conversations, which were meant to elicit a negative and a positive interaction context. Based on participants' continuous reports of the valence of their emotional experience, we looked for changes in three statistical measures, aiming to capture emotional similarity between partners (i.e., does their valence fluctuate together). Specifically, we investigated the occurrence, frequency and direction of change in partners' linear correlations, instantaneous derivative matching (IMD) and signal matching (SM). While correlation changes were only observed in $2 \%$ of the couples, IDM changes were detected for about one third of the couples (34\%), and SM changes were detected in about half of them (49\%). Most couples demonstrated one change point, and the direction of the change differed depending on the specific emotional similarity measure. In a first validation of this method, we demonstrated how such change points can pinpoint to subtle, but meaningful dynamic processes in couples. We end by discussing the added value of change point detection analyses for relationship research and interpersonal research in general.
\end{abstract}




\section{Introducing Change Point Detection Analysis in Relationship Research: an Investigation of Couple's Emotion Dynamics}

The unique dynamics between romantic partners shape the quality and course of their intimate relationship. For instance, partners continuously elicit, respond to, and regulate emotional experiences in each other, and these interpersonal emotion dynamics are thought to be at the core of intimate relationships (Schoebi \& Randall, 2015). They predict not only the longevity of the relationship (Gottman, 2014), but also both partners' physical (Sbarra \& Coan, 2018) and mental health (Hofmann, 2014).

Although posited in many theories and supported by research, studying such interpersonal emotion dynamics is inherently challenging. One particular challenge is that relationship partners shape each other's emotional experiences in different ways, and different emotion dynamics can thus be investigated (e.g., Schoebi \& Randall, 2018). Even a specific concept such as emotional similarity can be investigated in different ways across time. One can look at (1) similarities in how partners' emotional experiences fluctuate throughout time (shape similarity), (2) similarities in the variance of these experiences (scatter similarity), or (3) similarities in the average intensity of their experiences (although less dynamic; level similarity) (Furr, 2010) ${ }^{1}$. Throughout an interaction, partners can be highly emotionally similar in the sense that they simultaneously start feeling more positive or negative, and thus show similar emotional changes (high shape similarity); while they can be highly dissimilar in the sense that one partner is consistently feeling much more positive than the other one (low level similarity).

A second challenge is that, rather than staying constant, partners' interpersonal emotion dynamics are expected to change over time themselves, to match different contexts

\footnotetext{
${ }^{1}$ These different aspects of similarity are especially applied and known in fields of relationship science, such as personality similarity research (see e.g., Furler, Gomez, \& Grob, 2013).
} 
or goals (Butler, 2017). These changes can be abrupt, implying non-linear processes. Coming back to the example of emotional similarity, couples' emotional experiences are more likely to be similar to each other if partners are watching a movie together than when they are at their separate workplaces.

To capture meaningful changes in interpersonal emotion dynamics, methods are needed that identify change points in a wide variety of interpersonal dynamics, regardless of how the specific dynamic was operationalized. Up till now, such methods were missing. To fill this gap, this paper introduces a change point detection method that was recently developed to study within-person changes in dynamics (Cabrieto et al., 2019). We advocate that it can be a useful tool for studying interpersonal (emotion) dynamics as well. We introduce its use by investigating change in three different aspects of emotional similarity (i.e., shape, scatter, level) between partners. By allowing to capture how dynamics between partners change over time, this change point detection approach can advance our micro-level insight into relationship functioning.

Below, we first discuss the domain of interpersonal emotion dynamics more elaborately, and the data that is needed to study changes in interpersonal dynamics. Next, we review the statistical methods that are currently used to this end. We distinguish between model-based and more descriptive approaches, and build the case for our change point detection approach.

\section{The investigation of interpersonal emotion dynamics}

In its broadest sense, the term interpersonal emotion dynamics captures the myriad forms of interconnection that arise between partners' behavior and emotional experiences across time (Sels et al., 2021). In a more strict sense, it captures the different connections that arise between partners' emotions (Randall \& Schoebi, 2018). For instance, partners can elicit frustration in each other when they feel hampered in their goals, but also sadness, when one 
feels sympathy for something that occurred to the partner (Keltner \& Haidt, 1999). As another example, partners can carry over stress to each other (Thompson \& Bolger, 1999), they can try to consciously regulate each other's worries and negative emotions (Dixon-Gordon et al., 2015), or they can calibrate their emotions during communal activities (Parkinson, 2020).

Depending on the emotion researcher's interest, different dynamics can thus be investigated, focusing on specific emotions and processes that all provide unique information about the couple's functioning. This also includes specific temporal and statistical patterns. As we mentioned above, the concept of emotional similarity can be captured by looking at shape, scatter, or level similarity, which all imply different statistical measures (Furr, 2010). As another example, partners' emotions can start to fluctuate together during an interaction, their emotions can start to predict each other, or their emotions can become coupled with each other over time (Estrada et al., 2020). As a result, different statistical methods have been used and proposed to capture interpersonal emotion dynamics, both linear and nonlinear (Estrada et al., in press).

An additional complexity is that the influences that partners exert on each other's emotions are not expected to be constant, but dynamic (Butler, 2017). In some contexts, partners would strongly influence each other's emotions, resulting in strong interpersonal emotion linkages, and in others, their emotions change independently (e.g., Butler \& Randall, 2013). While intuitive, such changes in interpersonal emotion dynamics have mostly been ignored in current literature, probably because statistical methods for capturing them were still under development.

Interpersonal emotion dynamic research would thus benefit from flexible methods that capture meaningful changes in different interpersonal emotion dynamics. Indeed, advancing understanding on when and how emotions between persons become linked to each other, has been called an important future step for this field (Randall \& Schoebi, 2018). 


\section{What kind of data do we need?}

Investigating interpersonal dynamics requires intensive longitudinal data (Gable \& Reis, 2005). The psychological variables of interest, such as partners' emotions, have to be sampled at a high frequency in order to capture the couple's changes in interpersonal dynamics over time (e.g., Butler, 2017). For instance, standardized dyadic laboratory interactions can be used, in which couples interact, and both partners afterwards report on their emotional experiences throughout the interaction by video-mediated recall (Roberts et al., 2007). Other measures capture real time emotional facial and vocal expressions, and peripheral and central physiology (Calvo et al., 2015).

This sort of data allows us to detect changes in interpersonal dynamics, and if such changes can be identified, additional information about the context or interaction is needed to uncover its meanings. In other words, what happens when partners' emotions for example start to become more or less similar?

\section{How can we capture interpersonal dynamics?}

Model-based approaches are a first family of statistical tools to capture interpersonal dynamics. This family is diverse, with different statistical models implying strongly different theoretical assumptions about how dynamics unfold (Gates \& Liu, 2016). A distinction is made between linear and nonlinear approaches. Linear approaches such as autoregressive models, dynamic factor analysis and actor-partner independence models are extensions of (multilevel) regression approaches, accounting for the dependencies between partners and across time (i.e., scores adjacent in time likely correlate). For emotions, such statistical models can for instance capture emotional similarity between partners by modelling the concurrent covariation between these partners' emotions over time.

There is also increasing attention for nonlinear models in relationship science, which involve nonlinear patterns such as cyclical rhythms (Gable \& Reis, 2005; Girme, 2020). In the 
case of interpersonal emotion dynamics, this for instance means that one assumes that emotions tend to follow cyclical, oscillatory patterns, and that partners may exert influences on each other's emotional cycles (Butler \& Barnard, 2019) or may be emotionally similar in that they oscillate together. Such emotion dynamics can be captured by coupled oscillator models (Butner et al., 2005) or coupled nonlinear difference equations (Gottman et al., 2002).

Most statistical models assume stationarity, implying that the dynamics remain constant over time. As argued above, this assumption often unsustainable because interpersonal emotion dynamics are expected to change over time. Increasing attention is therefore paid to model extensions that allow the dynamics to change themselves, but that again have their own theoretical and statistical assumptions (for within-person modeling approaches capturing change in dynamics, see Ariens et al., 2020).

Next to model-based approaches, researchers use descriptive statistical measures to capture relevant dynamics, that pertain to linear or nonlinear processes (e.g., Ferrer, 2016). For instance, the extent to which partners' emotions covary together or predict each other over time, can be captured by (cross-concurrent or time-lagged) correlations (e.g., Sels et al, 2019). Other descriptive statistical measures are more graphical, and rely on calculating how long couples remain in or how often they return to a certain emotional state (e.g., Butler et al., 2014). For instance, emotional similarity can be operationalized by correlations, mainly capturing the extent to which partners' emotions fluctuate together (shape similarity), or difference measures, also capturing other aspects of similarity (scatter and/or level similarity).

Interestingly, one could use such descriptive approaches to discover if and which interpersonal emotion dynamics change across time, to account for non-stationarity. In the case of emotional similarity, it could thus be captured if and how partners' become more or less similar in how their emotional experiences fluctuate (changes in shape similarity), or in whether average emotional experiences or emotion variances become more similar or diverge 
more throughout time (changes in level similarity or scatter similarity). Up to now, there were no statistical methods to do this.

\section{KCP-RS Change Point Detection Analysis}

The KCP-RS change point detection method allows to identify abrupt changes in descriptive statistical measures and thus interpersonal dynamics. Dependent on one's theory and interpersonal focus, different statistical measures can be chosen and implemented to investigate longer-lasting changes in them. For instance, if one is interested in concurrent linkages between partners' emotions, correlations can be implemented (assessing linear relationships), but distance correlations (assessing both linear and nonlinear relationships) can be implemented as well (Sels et al., 2019). KCP-RS divides the intensive longitudinal data into a few phases of adjacent time points. Within a phase, the statistic of interest remains relatively constant, whereas the transition to a next phase indicates an increase or decrease in this statistic. Change point detection methods thus provide the tools to verify empirically if hypothesized changes (both linear and nonlinear) in interpersonal dynamics can be found. The flexibility of KCP-RS regarding the statistic implemented is particularly relevant for domains in relationship science such as the field of interpersonal emotion dynamics, where different dynamics are of interest.

\section{Present study}

With this study, we aim to showcase the KCP-RS change point detection method as a useful tool for investigating interpersonal dynamics in intimate relationships. We focus on couples' interpersonal emotion dynamics during standardized interaction tasks, and on one specific form of interpersonal dynamics: similarity in partner's emotional valences.

We investigate valence, because in several influential emotion theories, valence and arousal have been considered the two fundamental dimensions of emotions (Barrett \& 
Russell, 1998; Russell, 2003). While arousal captures people's activation, ranging from low to high, valence captures a subjective feeling of (un)pleasantness, ranging from pleasure to displeasure. Increasingly, especially valence is considered the building block of emotional life (Barrett, 2006), with research showing the relative importance of valence over arousal (Kron, et al., 2015).

Further, valence can be assessed continuously by an affect rating dial (Ruef \& Levenson, 2007). This established measure allows to obtain intensive sampling of people's emotional experiences without putting too much load on people, and captures the breadth of emotions. Specifically, in our study, after couples engaged in two different conversations (meant to elicit a negative and a positive interaction), both partners watched recordings of the interactions and indicated their momentary emotional experiences, resulting in second-bysecond self-reports of valence.

Using these data, we aim to identify changes in three statistical measures that capture emotional similarity between partners. Specifically, we focused on: (1) partners' correlations, (2) instantaneous derivative matching (IDM), and (3) signal matching (SM). The first measure captures linear relationships between partners' emotional experiences, IDM captures mean differences in both partners' rates of change across time, and SM captures the mean absolute difference between both partners' valence scores. These measures differ in which aspects of emotional similarity they are sensitive to. While the correlation is only sensitive to shape similarity, IDM also captures scatter similarity, and on top of that, SM takes into account level similarity as well. When partners' emotional experiences evolve in a similar way over an interaction, this will thus result in a high correlation, regardless of possible scatter or level differences. Instead, the resulting SM scores will depend on all three components and will only signal strong similarity if all three aspects are similar. The meaning of changes in these three measures thus also differ. We want to stress that any descriptive statistic, meant to 
capture interpersonal (emotion) dynamics can be implemented, which is an important benefit of the proposed method.

\section{Method}

\section{Participants, procedure, and materials}

Participants were recruited for a larger study on emotion dynamics in intimate relationships (see https://osf.io/ra8jf/ for publications on these data. A description of the full dataset can be found on the EMOTE database). They were recruited through social media platforms, and flyers and posters that were distributed in Leuven, Belgium. The final sample included 101 couples that self-identified as heterosexual, who were on average 26 years old $(\mathrm{SD}=5$ years, $\mathrm{Min}=18, \mathrm{Max}=53)$, and had been in a relationship for 4.5 years $(\mathrm{SD}=2.8$, $\min =7$ months, $\max =21$ years $).$ The majority of these couples was living together $(n=96)$ and did not have children yet $(\mathrm{n}=5)$.

The nationality of most participants was Belgian $(n=187)$. The other participants had

a Dutch $(n=9)$, German $(n=3)$, Armenian $(n=1)$, Chinese $(n=1)$, or Ukrainian nationality $(n=1)$. Among half of the participants $(n=102)$ had a University degree, one fourth had completed higher education $(n=43)$, and the remainder had a primary school $(n=1)$ or secondary school education level $(\mathrm{n}=56)$. The study was approved by the ethics committee of the Faculty of Psychology and Educational Sciences of the KU Leuven.

The full study consisted of different parts: an online pre-lab assessment, a lab part, an experience sampling part, and two follow-ups. For the present study, only the lab part is relevant. Not all couples provided complete data for the lab portion of the study, resulting in a sample of 96 couples.

After filling out pre-lab questionnaires online, participants were invited to the lab. Here, they participated in three different conversations with each other. First, participants 
discussed a neutral topic for two minutes, which involved the events of the previous day. Next, participants discussed for ten minutes what they regarded as each other's most annoying characteristic, a topic that was meant to elicit a negative interaction. Finally, participants discussed for ten minutes what they regarded as each other's most valuable characteristic, a topic that was meant to elicit a positive interaction. To facilitate these conversations, each partner individually reported, in a separate room and before each interaction, on his/her partner's most annoying and most valuable characteristic. When they returned to the common room, a research assistant asked to discuss both partners' selected characteristic during the following conversation. Couples were told that they could choose who would begin the conversation, and that they would be notified after 8 minutes to finish up the conversation, aiming to end on good terms. This notification will be referred to as the 'knock on the door'. After the research assistant left, the conversation started. After every conversation, both partners filled in questions that assessed their experience of the corresponding conversation in separate rooms. Afterwards, participants separately engaged in video-mediated recall (Roberts et al., 2007). Partners watched the conversation on the negative and positive topics and continuously rated their emotional experience, by moving a joystick to the left $(-1=$ very negative) and the right ( 1 = very positive). This procedure yielded second-to-second scores of their emotional experience in terms of valence.

\section{Data-analyses: Choice of running statistics}

To measure emotional similarity, we focus on correlation, signal matching, and instantaneous derivative matching, which differ in whether or not they are sensitive to shape similarity, scatter similarity or level similarity. The correlation $r$ indicates the strength of the linear relationship between the observed scores of both partners. A higher $r$ thus indicates stronger emotional similarity. 
The instantaneous derivative matching measure (Elkins et al., 2009) captures similarity in terms of slopes. First, for each partner, the slopes are obtained by calculating the difference in scores between each time point $t$ and the previous time point $t-1$. Next, the slopes of the two partners are compared for each $t$, by calculating the absolute difference. The IDM equals the average slope difference:

$$
I D M=\frac{1}{T} \sum_{t=1}^{T}\left|\left(a_{t-1}-a_{t}\right)-\left(b_{t-1}-b_{t}\right)\right| .
$$

$a_{t}$ and $b_{t}$ denote the score at time point $t$ of partner 1 and partner 2, respectively, where $t=$ $[1, \ldots, T]$. The larger the slope differences, the higher the IDM value, indicating lower similarity. In case the partners are perfectly similar, IDM is equal to 0.

Finally, signal matching (Elkins et al., 2009) captures similarity by evaluating the differences in the scores of both partners across time. At each time point $t$, the absolute difference in scores of the partners is calculated. The SM then equals the average of the absolute differences:

$$
S M=\frac{1}{T} \sum_{t=1}^{T}\left|a_{t}-b_{t}\right|
$$

Similar to IDM, a higher SM value represents lower emotional similarity.

Figure 1 illustrates the impact of changes in shape, scatter and level of partners' emotional experiences on $r$, IDM and SM. Each panel shows hypothetical emotion scores for two partners across time. Orange scores represent one partner, and blue scores the other one. Left of the vertical black line, partner scores are identical in terms of shape and scatter, and 
there is an average level difference of 1 . One partner thus consistently scores 1 point higher on its emotional experience than the other partner. Here, $r=1$, IDM $=0$ and $\mathrm{SM}=1$. On the right side of the line, a change in shape, scatter, level, or a combination in the scores of one the partners is introduced, and the impact of the type of change on $r$, IDM and SM is shown. An example of a shape change is shown in Figure 1a. The shape change leads to a decrease in $r$ (1 to .25), and an increase in IDM (0 to 3 ) and SM (1 to 1.8). Figure $1 \mathrm{~b}$ illustrates a scatter change, where the variance of the orange scores increases from 3 on the left to 27 on the right. This leads to an increase in IDM (0 to 5) and SM (1 to 2.2), whereas $r$ remains the same (as it is insensitive to scatter changes). Figure 1c displays a level change, where the mean of the orange scores increases from 0 on the left to 1 on the right. This results in an increase in SM (from 1 to 2), whereas the other measures are unaffected. These patterns generalize to situations in which combinations of these changes are introduced. Changes in shape and scatter (Figure 1d), as well as changes in shape and level (Figure 1e), influence all three measures. Changes in scatter and level (Figure 1f) impact IDM and SM. Finally, changes in shape, scatter and level (Figure 1g) impact all three measures. Thus, $r$ is sensitive to changes in shape, IDM is sensitive to changes in both shape and scatter, and SM is sensitive to changes in shape, scatter and level.

We performed additional KCP-RS analyses for the mean and variance, which are available in the R package kcpRS, together with the correlation (Cabrieto et al., 2021). These analyses showcase how the method can be used for different measures, and are reported in the supplemental material available at https://osf.io/ra8jf/. Additional code was written for signal matching and instantaneous derivative matching, and an example of $\mathrm{R}$ code and corresponding output can be found at https://osf.io/ra8jf/.

\section{Data-analyses: KCP-RS}


The KCP-RS analysis consists of four steps that we will briefly explain below and illustrate by the data of one couple. We conduct the steps for $r$, IDM, and SM.

\section{Step 1: Deriving the running statistics}

The main goal of KCP-RS analysis is to detect changes in a specific statistic. The first step is to derive a time series of running values of this statistic, that is, the short-term values of this statistic across time. These running values are obtained by sliding a window along the time series of the observed data, and computing the statistic of interest in each window. The size of the window (i.e., number of time points used to compute the statistic) can be set by the user. Based on simulation studies (Cabrieto et al., 2018a), the default window size is 25 time points, thus 25 seconds in our example. However, as we will showcase below, we recommend the user to run the KCP-RS analysis with different window sizes to check the robustness of the results in terms of the detected change points.

For our example couple, Figure 2 a shows the raw bivariate data and Figure $2 \mathrm{~b}$ displays the running $r$ s, IDMs, and SMs which were calculated using a window size of 25 seconds (resulting in $1200-25+1=1176$ windows). As for all the couples, the data start with the negative interaction context, and the transition between the interaction contexts is located at 600 seconds. The negative and positive interaction contexts thus consist of seconds 1-600 and seconds 601-1200, respectively. Inspecting Figure 2b, the fluctuations in the running $r$ s and IDMs do not show a longer-lasting change, whereas the SM level seems to increase for about four minutes during the positive interaction context.

\section{Step 2: Locating possible change points in the running statistics}

Next, the analysis is run $K_{\max }$ times with the number of considered change points $K$ ranging from 0 to $K_{\max }=10$. For each $K$-value, the time series of running statistics is optimally divided into $K+1$ distinct phases. Optimally delineated phases are characterized by 
level changes in the statistic of interest, whereas the running values within a phase are as homogenous as possible. The homogeneity $\hat{R}_{\min , K}$ of the running values within the $K+1$ phases is quantified using a Gaussian kernel function.

Table 1 shows the $\hat{R}_{\min , K}$ values and change point locations for the running SMs of couple 1 . The $\hat{R}_{\min , K}$ values improve (i.e., decrease, indicating more homogenous phases) when more change points are allowed (i.e., when $K$ increases). If we consider the different rows of Table 1 (i.e., the different $K$ values), we see in the columns (CP1, CP2, etc.) that we consistently find change points in the positive interaction context (between seconds 6011200), one located briefly after the transition and the other located in the middle (i.e., always between 675 and 677 seconds, and between 871 and 899 seconds).

\section{Step 3: Choosing the number of change points}

Next, a significance test is conducted to establish whether the statistic changes at least once across the time series. If so, a grid search is performed to determine the optimal number of change points.

Significance test. The permutation-based significance test is called the variance drop test (Cabrieto, 2018a), as it looks at the largest drop in the $\hat{R}_{\min , K}$ values when allowing for an additional change point, and compares this improvement to that observed in a large number (i.e., 1000 by default) of permuted data sets. These permuted data are obtained by randomly shuffling the time points in the original data, and afterwards recomputing the change point locations and $\hat{R}_{\min , K}$ values. The test builds on the idea that if there is at least one change point present in the running statistics for the observed data, the improvement in $\hat{R}_{m i n, K}$ will be large for at least one considered $K$ value. If there is no clear change point in these running statistics, the small improvement will be comparable to that for the permuted data. The test

yields a $p$-value by computing the proportion of permutations in which the largest $\hat{R}_{\min , K}$ drop 
of the permuted data was larger than the largest $\widehat{R}_{\min , K}$ drop of the observed data, divided by the total number of permutations. The obtained $p$-value is then compared to a prespecified significance level. When screening for changes in multiple statistics, the overall type 1 error rate is controlled for by applying a correction to the overall significance level $\alpha$. The Bonferroni correction is applied by default, implying that each statistic of interest will be assigned a significance level of $\alpha$ divided by the number of tracked statistics.

Figure 2c shows that the largest drop in the $\hat{R}_{\min , K}$ curve for the running SMs of the observed data (blue line) is indeed larger than the largest $\hat{R}_{\min , K}$ drops for the permuted data ( $p$-value of 0 , with $\alpha=0.05 / 3)^{2}$. This means that there is at least one change point present in the SM level of our example couple. For the running $r$ s and IDMs, the $\hat{R}_{\min , K}$ curve of the observed data is similar to the $\hat{R}_{\min , K}$ curves of the permuted data sets, indicating that no correlation or IDM change point are present in the data.

Grid search to decide on the exact number of change points. Cabrieto et al. (2018a) built on the proposal of Arlot et al. (2012), who added a penalization term to $\hat{R}_{\min , K}$ to, account for the number of change points: $\hat{R}_{\min , K}+C *$ penalty. Allowing for more change points is thus only warranted if it lowers $\hat{R}_{\min , K}$ after compensating for the increased penalty. The penalty coefficient $C$ determines the weight of the penalty term: large $C$ values will lead to a low number of change points, and vice versa. This penalty coefficient can be tuned through a grid search (Cabrieto et al., 2017), that considers a large range of possible $C$ values. In the end, the most frequently returned number of change points across all considered coefficient values is selected $(K)$. The change point locations associated with $K$ represent the final change point solution for that statistic.

\footnotetext{
${ }^{2}$ As we are focusing on the three interpersonal dynamics measures ( $r$, SM and IDM), $\alpha=0.05 / 3$. However, $\alpha$ can also be divided by 5 for all measures (including mean and variance), which slightly decreases the number of detected change points.
} 
For our example couple, value 2 was most frequently returned by the grid search (see Figure 2), corresponding to two SM changes at 676 and 873 seconds (see Table 1).

\section{Step 4: Checking the robustness of the located change points (optional)}

Finally, the user can check whether the results are similar across different window sizes. If so, one can be more confident about the presence as well as the location of the change points. For our example couple, the KCP-RS analysis with a window size of 25 resulted in two SM changes at 676 and 873 seconds. Using alternative window sizes between 10 and 30 also consistently resulted in two detected SM change points. The first change point location ranged from 675 to 677 , and the second change point location ranged from 871 to 876 , indicating that the findings are robust. No change points were detected when window sizes 35 and 40 were used.

\section{Results}

We first provide a summary of the results across couples. Next, we zoom in on our example couple, and interpret the detected change points in this couple by linking them to the couple's observed interaction behavior.

\section{Overall number and timing of $r$, IDM, and SM change points}

Figure 3 shows the detected $r$, IDM and SM change points per couple ${ }^{3}$. Table 2 summarizes these results by showing the number of change points found over all couples, as

\footnotetext{
${ }^{3}$ We also trimmed the interaction data, removing parts that might not reflect couples' natural dynamics. We trimmed the first 30s of both interactions. We also trimmed the first 30s and the last $120 \mathrm{~s}$, to discard the period after the knock on the door. Furthermore, we investigated whether lagging the data of one of the partners influenced the results, as partners may not change simultaneously. Based on previous research, we took a lag of 8s (Sels et al., 2020). Trimming the first 30s and lagging the data did not alter the results substantially, in terms of number of couples with change points and total number of change points (see https://osf.io/ra8jf/). Slightly different results were obtained when using the more extensively trimmed data (first 30s and last 120s). Overall, less couples had change points and less overall change points were detected. This makes sense because we discarding the knock on the door part excluded a possible source of
} 
well as their location (negative context, transition period, positive context). Whereas $r$ changes were rare (only 2 couples; $2 \%$ ), IDM changes were detected in about one third of the couples (33 couples; 34\%), and SM changes in about half of them (47 couples; 49\%). The concrete operationalization of emotional similarity thus had a major impact on the obtained results.

The majority of the couples with an IDM or SM change showed only one change point. Twenty-five, 3, 4 and 1 couple(s) had 1, 2, 3 and 6 IDM change points. Twenty-six, 11, 7, 2 and 1 couple(s) had 1,2, 3, 4 and 5 SM change points. Only a small fraction of the change points (ranging from 0 to $14 \%$ ) were related to the transition, whereas the other ones were evenly spread across the negative and positive interaction contexts.

IDM levels were most often lower after the change point $(76 \%$ in the negative and $71 \%$ in the positive interaction context), indicating that partners became more similar in terms of IDM throughout both interactions. For SM change points, the direction differed depending on the interaction context. SM levels were often lower after the change point in the negative interaction context (67\%), indicating that partners became more emotionally similar in terms of SM. In the positive interaction context, SM levels were most often higher after the change point (54\%), indicating a lower emotional similarity in terms of SM throughout this interaction.

\section{What can change points in emotion dynamics tell us?}

To further understand the meaning of identified change points in couples' emotion dynamics, we returned to our example couple (see Figure 2) and related the detected change points in emotional similarity to what actually happened during the couple's conversation. As

similarity changes, and the time series lengths were reduced by $25 \%$, which reduced the power to pick up changes. 
stated above (see also Figure 4), the couple had two SM change points in the positive interaction context, and the description is thus focused on this context only.

At the start of the positive conversation, the female partner (Indiv2) briefly described the most valuable characteristics of her partner ("being good with children"). Around that time, the male partner (Indiv1) showed a positive valence rating. Next, they switched roles (around second 664), and the male partner started to describe his partner's most valuable characteristic: being helpful ("whenever there is a problem, she is the first to offer help or to address this problem"). In describing this characteristic, some subtle ambiguities could be noticed (both verbally and in the man's written report), that might had been overlooked if they had not corresponded with the first identified change point (at second 676). Although the male partner stated the praiseworthiness of the characteristic, he also noted some disadvantages. For example, he mentioned that this characteristic is not always appreciated by everyone, and that he would not intervene in situations as much as his partner does. His ambiguity seemed to be reflected in his valence ratings, which became a bit more negative. His partner was clearly convinced of the positivity of the characteristic, and her valence ratings remained positive. This discrepancy seemed to result in the first SM change point, where there was an increase in SM, indicating lower inter-partner similarity in their average emotional experience or level similarity (see Figure 2b).

When the couple switched topics (around second 902) and started focusing on the male partner's valuable characteristic ("how attractive she finds it with regards to future prospects if a man is good with children"), the male partner's valence ratings increased again. This moment corresponded with the second SM change point (at second 873, see Figure $2 b$ ), at which both partner's valence ratings were high. Stronger similarity in their average emotional experience (or level similarity) was thus reflected in an SM decrease.

\section{Discussion}


Up to now, changes in interpersonal dynamics across time have been largely neglected. This is an important gap in current research, as it is highly realistic that changes in interactions will alter at least some of the dynamics taking place between partners, such as their emotion dynamics. To allow researchers to capture and investigate such changes in interpersonal dynamics, we presented the change point detection method KCP-RS. Next to intimate relationships, it can be used in interpersonal contexts such as parent-child interactions, friendships, and any other interaction between two persons.

For the domain of interpersonal emotion dynamics specifically, the availability of such a change point detection method can constitute important progress. People's emotions are expected to change continuously, to emerge and to disappear again, and to link and unlink between persons over time. By allowing to detect these on-and offsets, change point detection methods address an important barrier to interpersonal emotion dynamic research (Butler, 2015).

To illustrate KCP-RS, we used data of 96 couples who engaged in two conversations, meant to elicit a negative and a positive interaction context. Based on participants' selfreported valence fluctuations, we identified changes in three statistical measures that reflected different aspects of emotional similarity, a specific form of interpersonal emotion dynamics. Specifically, we investigated the occurrence, number and direction of changes in partners' linear correlations, signal matching and instantaneous derivative matching. Our findings indicated that correlation changes were rare, meaning that most partners did not change substantially in how much their emotional experiences fluctuated together (shape similarity). This finding is notable as studies on interpersonal emotion dynamics often focus on linear relationships between partners' emotional experiences. One explanation is that many couples show no correlations in their self-reported emotions to begin with. Indeed, previous 
research with these sorts of interactions and samples has shown that most partners' emotions do not substantially correlate (Sels, et al., 2019).

IMD changes were detected in about one third of the couples, and SM changes in about half of the couples. The higher number of change points can be explained by the fact that IDM and SM take more similarity aspects into account. IDM accounts for scatter similarity on top of shape similarity, whereas SM is additionally impacted by changes in level similarity. Capturing emotional similarity more comprehensively thus resulted in more detected changes. Changes in these measures can also tell us different things about couples' functioning. A higher correlation might be indicative for attunement (partners' emotions fluctuating more or less together), while a higher IDM and SM can also indicate more reactive responding of partners towards each other. In the field of personality psychology, it has already been shown that personality similarity measures that are differentially sensitive for shape, scatter and level, sometimes show different associations with marital quality (Wang et al., 2018). Our results thus demonstrate how much the measure that researchers select to capture interpersonal emotion dynamics matters, and signifies the importance of carefully paying attention to the concrete operationalization of a specific theory or hypothesis about dynamics.

In a preliminary validation of this method, we demonstrated that KCP-RS can detect subtle, but meaningful dynamic changes that might otherwise get overlooked. A qualitative description of one couple revealed that the detected change points in SM levels corresponded to ambiguities in the male partner's emotional experience. In general, the results highlight the utility of using change point detection methods to identify potentially meaningful changes in interpersonal dynamics throughout couple interactions. If it had been for instance assumed -as is done in many statistical models assuming stationarity- that every couple's emotional level was stable throughout the interaction, many couples had been inaccurately represented. 


\section{Future research}

Reflecting on the lack of correlation change points, one might conjecture that our interaction contexts were not strong enough, resulting in a low potential to elicit changes in dynamics. However, it is worth noticing that most couples showed substantial changes in their mean level of emotional experience in line with the changing interaction context.

Nevertheless, future studies should assess change points in contexts that more strongly elicit different sorts of emotions. Our sample also consisted of couples who were satisfied about their relationship, a sentiment potentially buffering against or overruling the kind of situationally and lab-based induced negativity we used in our study. In general, our sample consisted of a rather homogenous (although some demographic information was not assessed) and heterosexual sample, and it remains to be seen if results generalize to other samples. Further, assessing emotions as multi-componential constructs, by complementing self-reports measures with physiology and observed expressions (e.g., Scherer, 2009), might be an avenue worth exploring. The KCP-RS method has shown to gain power with an increasing amount of variables (Cabrieto et al., 2018b) and self-report measures are limited by individual biases and interoceptive accuracy (Paulhus \& Vazire, 2007), unlike observed expressions and physiology.

Additionally, we selected three descriptive statistical measures capturing emotional similarity to illustrate the method. Other dynamics could be investigated as well, such as cross-lagged correlations between partners' emotional experiences. These statistical measures can easily be implemented in the kcpRS package. Change point detection analysis merely provides an important tool to empirically investigate if changes in interpersonal dynamics that are hypothesized by emotion and relationship theories- are actually present in couple's interactions. 
For instance, an interesting question for future research is if the flexibility with which couples show emotional linkages matters (see also Sels et al., 2018). According to family system theories, who are very influential in clinical practice but scarcely empirically verified, functional couples would be responsive to their environments (Goldenberg \& Goldenberg, 2012). This implies that partners can be flexible in responding to, and connecting and disconnecting emotionally with each other. In this regard, Luginbuehl and Schoebi (2020) recently showed that partners of individuals whose emotions were very resistant to change (or not resistant to change at all), perceived their partners as less responsive, resulting in a decline in relationship satisfaction. Change point detection analysis could build further on this sort of reseach, by identifying if the extent to which partners show changes in their interpersonal emotion dynamics relates to important relationship processes and outcomes.

Indeed, an important future challenge is to further uncover what the obtained change points can tell us about the functioning of relationships. Unfortunately, except for the change from a negative to positive context, there was little contextual or other time-varying information available in the data, except for the videotaped interactions themselves. By describing this interaction, we aimed to tentatively provide some information, but in future research, videos could be coded on diagnostic and clinically relevant interaction patterns, such as demand-withdrawal patterns (Schrodt et al., 2014) or speaker/listener patterns, and it could be assessed if these correspond with observed change points.

Change point detection analysis on couple dynamics might be especially suitable for research on mechanisms of change, or so-called "turning points" in couple therapy. For instance, in emotionally focused couple therapy, change is expected to occur through the occurrences of new adaptive emotional experiences (Johnson, 2005). An important change event is "softening", which starts off by the experience and expression of vulnerable emotions by a usually hostile partner, after which a new form of emotional engagement is possible. By 
the same token, interrupting a couple's problem-maintaining interaction cycles is an important mechanism of change in many couple therapy approaches (Gurman, 2015). Change point detection analysis can enhance empirical support for couple interventions and therapy protocols.

Ultimately, change point detection analysis could help in identifying early warning signals of relationship problems and break-up, by automatically identifying meaningful changes in important processes that predict break-up. This is still a largely unexplored area in relationship research, but research in the psychopathology domain is increasingly identifying early warning signals such as emotional changes that precede the onset of psychopathology (Wichers et al., 2016).

\section{Conclusion}

This paper introduced change point detection analysis in relationship research, and demonstrated its utility for investigating couple dynamics. By re-analyzing couples' interaction data, we showed how KCP-RS can be applied to detect change in couples' emotion dynamics. Importantly, this method might also advance other areas of interpersonal relationship research. 


\section{References}

Ariens, S., Ceulemans, E., \& Adolf, K. (2020). Time series analysis of intensive longitudinal data in psychosomatic research: a methodological overview. Journal of Psychosomatic Research, 137, 1-15. doi:https://doi.org/10.1016/j.jpsychores.2020.110191

Arlot, S., Celisse, A., \& Harchaoui, Z. (2012). Kernel change-point detection. Opgehaald van https://arxiv.org/pdf/1202.3878v1.pdf

Barrett, F. L., \& Russell, A. J. (1998). Independence and bipolarity in the structure of current affect. Journal of personality and social psychology, 74(4), 967-984.

Barrett, L. F. (2006). Valence is a basic building block of emotional life. Journal of Research in Personality, 40(1), 35-55. doi:https://doi.org/10.1016/j.jrp.2005.08.006

Butler, E. A. (2015). Interpersonal affect dynamics: it takes two (and time) to tango. Emotion Review, 7(4), 336-341.

Butler, E. A. (2017). Emotions are temporal interpersonal systems. Current Opinion in Psychology, 17, 129-134. doi:https://doi.org/10.1016/j.copsyc.2017.07.005

Butler, E. A., \& Barnard, J. K. (2019). Quantifying interpersonal dynamics for studying socioemotional processes and adverse health behaviors. Psychosomatic Medicine, 81(8), 749-758.

Butler, E. A., \& Randall, A. K. (2013). Emotional coregulation in close relationships. Emotion Review, 5(2), 202-210. doi:https://doi.org/10.1177/1754073912451630

Butler, E., Hollenstein, T., Shoham, V., \& Rohrbaugh, M. J. (2014). A dynamic state-space analysis of interpersonal emotion regulation in couples who smoke. Journal of Social and Personal Relationships, 31(7). doi:https://doi.org/10.1177/0265407513508732

Butner, J., Amazeen, P. G., \& Mulvey, G. M. (2005). Multilevel modeling of two cyclical processes: extending differential structural equation modeling to nonlinear coupled systems. Psychological Methods, 10(2), 159-177.

Cabrieto, J., Adolf, J. K., Tuerlinckx, F., Kuppens, P., \& Ceulemans, E. (2018b). Detecting long-lived autodependency changes in a multivariate system via change point detection and regime switching models. Scientific Reports, 8(1), 1-15. doi:https://doi.org/10.1038/s41598-018-33819-8

Cabrieto, J., Adolf, J. K., Tuerlinckx, F., Kuppens, P., \& Ceulemans, E. (2019). An objective, comprehensive, and flexible statistical framework for detecting early warning signs of mental health problems. Psychotherapy and Psychosomatics, 88(3), 184-186. doi:https://doi.org/10.1159/000494356

Cabrieto, J., Meers, K., Schat, E., Adolf, J. K., Kuppens, P., Tuerlinckx, F., \& Ceulemans, E. (2021). kcpRS: An R package for performing kernel change point detection on the running statistics of multivariate time series. Behavior Research Methods, 1-22. doi:https://doi.org/10.3758/s13428-021-01603-8

Cabrieto, J., Tuerlinckx, F., Kuppens, P., Wilhelm, F. H., Liedlgruber, M., \& Ceulemans, E. (2018a). Capturing correlation changes by applying kernel change point detection on 
the running correlations. Information Sciences, 447, 117-139. doi:https://doi.org/10.1016/j.ins.2018.03.010

Calvo, R. A., D'Mello, S., Gratch, J. M., \& Kappas, A. (2015). The Oxford handbook of affective computing. Oxford Library of Psychology.

Dixon-Gordon, K. L., Bernecker, S. L., \& Christensen, K. (2015). Recent innovations in the field of interpersonal emotion regulation. Current Opinion in Psychology, 3, 36-42. doi:https://doi.org/10.1016/j.copsyc.2015.02.001

Elkins, A. N., Muth, E. R., Hoover, A. W., Walker, A. D., Carpenter, T. L., \& Switzer, F. S. (2009). Pjysiological compliance and team performance. Applied Ergonomics, 40(6), 997-1003. doi:http://doi.org/10.1016/j.apergo.2009.02.002

Estrada, E., Sbarra, D. A., \& Ferrer, E. (2020). Models for Dyadic Data. In A. Wright, \& M. N. Hallquist, Handbook of Research Methods in Clinical Psychology (pp. 350-368). Cambridge University Press.

Ferrer, E. (2016). Exploratory approaches for studying social interactions, dynamics, and multivariate processes in psychological science. Multivariate Behavioral Research, 51(2-3), 240-256. doi:10.1080/00273171.2016.1140629

Furr, M. R. (2010). The double-entry intraclass correlation as an index of profile similarity: meaning, limitations, and alternatives. Journal of Personality Assessment, 92(1), 1-15. doi:https://doi.org/10.1080/00223890903379134

Gable, S. L., \& Reis, H. T. (2005). Now and then, them and us, this and that: studying relationships across time, partner, context, and person. Personal Relationships, 6(4), 415-432. doi:https://doi-org.kuleuven.ezproxy.kuleuven.be/10.1111/j.14756811.1999.tb00201.x

Gates, K. M., \& Liu, S. (2016). Methods for quantifying patterns of dynamic interactions in dyads. Assessment, 23(4), 459-471. doi:https://doi.org/10.1177/1073191116641508

Girme, Y. (2020). Current Directions in Psychological Science. 2020, 29(4), 351-357. doi:https://doi.org/10.1177/0963721420920598

Goldenberg, H., \& Goldenberg, I. (2012). Family therapy: an overview. Canada: Cengage Learning.

Gottman, J. M. (2014). What predicts divorce? The relationship between marital processes and marital outcomes. Psychology Press.

Gottman, J. M., Coan, J., Carrere, S., \& Swanson, C. (1998). Predicting marital happiness and stability from newlywed interactions. Journal of Marriage and Family, 60(1), 5-22.

Gottman, J., Swanson, C., \& Swanson, K. (2002). A general systems theory of marriage: nonlinear difference equation modeling of marital interaction. Personality and Social Psychology Review, 6(4), 326-340. doi:https://doi.org/10.1207/S15327957PSPR0604_07

Gurman, A. S. (2015). Clinical Handbook of Couple Therapy. New York: the Guilford Press. 
Hofmann, S. G. (2014). Interpersonal emotion regulation model of mood and anxiety disorders. Cognitive therapy and research, 38(5), 438-492.

Hollenstein, T., \& Lewis, M. D. (2006). A state space analysis of emotion and flexibility in parent-child interactions. Emotion, 6(4), 656-662. doi:10.1037/1528-3542.6.4.656

Johnson, S. M. (2005). Emotionally Focused Couple Therapy. In A. S. Gurman, Clinical Handbook of Couple Therapy (pp. 107-138). New York: The Guilford Press.

Keltner, D., \& Haidt, J. (1999). Social functions of emotions at four levels of analysis. Cognition and Emotion, 13(5), 505-521.

Kron, B., Pilkiw, A., Banaei, M., Goldstein, J., Anderson, A., \& Keith, A. (2015). Are valence and arousal separable in emotional experience? Emotion, 15(1), 35-44.

Luginbuehl, T., \& Schoebi, D. (2018). Emotion dynamics and emotion regulation in intimate relationships. In P. M. Cole, \& T. Hollenstein, Emotion regulation: a matter of time (pp. 208-225). New York: Routledge.

Parkinson, B. (2020). Intragroup emotion convergence: beyond contagion and social appraisal. Personality and Social Psychology Review, 24(2). doi:https://doi.org/10.1177/1088868319882596

Paulhus, D. L., \& Vazire, S. (2007). The self-report method. In R. W. Robins, C. R. Fraley, \& R. F. Krueger, Handbook of research methods in Personality Psychology (pp. 224239). New York: The Guilford Press.

Pijeira-Diaz, H. J., Drachsler, H., Järvela, S., \& Kirschner, P. A. (2016). Investigating collaborative learning success with physiological coupling indices based on electrodermal activity. (pp. 64-73). Proceedings of the sixth international conference on learning analytics \& knowledge.

Randall, A. K., \& Schoebi, D. (2018). Interpersonal emotion dynamics in close relationships. New York: Cambridge University Press.

Roberts, N. A., Tsai, J. L., \& Coan, J. A. (2007). Emotion elicitation using dyadic interaction tasks. In J. A. Coan, \& j. J. Allen, Handbook of emotion elicitation and assessment (pp. 106-123). Oxford: Oxford University Press.

Ruef, A. M., \& Levenson, R. W. (2007). Continuous measurement of emotion. In J. A. Coan, \& J. B. Allen, Handbook of emotion elicitation and assessment (pp. 286-297). Oxford University Press.

Russell, J. A. (2003). Core affect and the psychological construction of emotion. Psychological Review, 110(1), 145-172.

Sbarra, D. A., \& Coan, J. A. (2018). Relationships and health: the critical role of affective science. Emotion Review, 10(1), 40-54. doi:https://doi.org/10.1177/1754073917696584

Scherer, K. R. (2009). The dynamic architecture of emotion: evidence for the component process model. Cognition and Emotion, 23(7), 1307-1351. 
Schneider, B., Dich, Y., \& Radu, I. (2020). Unpacking the relationship between existing and new measures of physiological synchrony and collaborative learning: A mixed methods study. International Journal of Computer-Supported Collaborative Learning, 15(1), 89-113. doi:https://doi.org/10.1007/s11412-020-09318-2

Schoebi, D., \& Randall, A. K. (2015). Emotional dynamics in intimate relationships. Emotion Review. doi:10.1177/1754073915590620

Schrodt, P., Witt, P., \& Shimkowski, J. R. (2014). A meta-analytical review of the demand/withdraw pattern of interaction and its associations with individual, relational, and communicative outcomes. Communication Monographs, 81(1), 28-58.

Sels, L., Cabrieto, J., Butler, E., Reis, H., Ceulemans, E., \& Kuppens, P. (2019). The occurrence and correlates of emotional interdependence in romantic relationships. Journal of Personality and Social Psychology.

Sels, L., Ceulemans, E., \& Kuppens, P. (2018). A general framework for capturing interpersonal emotion dynamics. In A. Randall, \& D. Schoebi, Interpersonal emotion dynamics in close relationships (pp. 27-46). New York: Cambridge University Press.

Thompson, A., \& Bolger, N. (1999). Emotional transmission in couples under stress. Journal of Marriage and Family, 61(1), 38-48. doi:10.2307/353881

Wang, S., Kim, K., \& Boerner, K. (2018). Personality similarity and marital quality among couples in later life. Personal Relationships, 25(4), 565-580. doi:https://doi.org/10.1111/pere.12260

Wichers, M., Groot, P. C., Psychosystems, E. S., \& EWS Group. (2016). Critical slowing down as a personalized early warning signal for depression. Psychotherapy and Psychosomatics, 85(2), 114-116. 
Table 1

$\widehat{R}_{\text {min,K }}$ Values and Optimal Change Points $(C P)$ When Screening the Running Signal Matching Scores of the Example Couple

\begin{tabular}{lllllllllllll}
\hline $\mathrm{K}$ & $\widehat{\mathrm{R}}_{\mathrm{min}, \mathrm{K}}$ & $\mathrm{CP} 1$ & $\mathrm{CP} 2$ & $\mathrm{CP} 3$ & $\mathrm{CP} 4$ & $\mathrm{CP} 5$ & $\mathrm{CP6}$ & $\mathrm{CP7}$ & $\mathrm{CP} 8$ & $\mathrm{CP9}$ & $\mathrm{CP} 10$ \\
\hline 0 & 0.4522 & 0 & 0 & 0 & 0 & 0 & 0 & 0 & 0 & 0 & 0 \\
1 & 0.4064 & 899 & 0 & 0 & 0 & 0 & 0 & 0 & 0 & 0 & 0 \\
2 & 0.343 & 676 & 873 & 0 & 0 & 0 & 0 & 0 & 0 & 0 & 0 \\
3 & 0.3222 & 327 & 677 & 873 & 0 & 0 & 0 & 0 & 0 & 0 & 0 \\
4 & 0.2978 & 327 & 530 & 675 & 873 & 0 & 0 & 0 & 0 & 0 & 0 \\
5 & 0.2803 & 37 & 327 & 530 & 675 & 873 & 0 & 0 & 0 & 0 & 0 \\
6 & 0.2633 & 364 & 409 & 494 & 526 & 675 & 873 & 0 & 0 & 0 & 0 \\
7 & 0.247 & 327 & 367 & 408 & 494 & 526 & 675 & 873 & 0 & 0 & 0 \\
8 & 0.2295 & 37 & 326 & 367 & 408 & 494 & 526 & 675 & 873 & 0 & 0 \\
9 & 0.2137 & 37 & 326 & 367 & 408 & 494 & 526 & 675 & 875 & 1049 & 0 \\
10 & 0.1991 & 37 & 326 & 367 & 408 & 494 & 526 & 675 & 871 & 907 & 1048 \\
\hline & & & & & & & & & & & \\
\hline
\end{tabular}

Note: The numbers in columns CP1 to CP10 represent the change point locations in seconds for the different numbers of considered change points K. Seconds 1-600 represent the negative interaction context, and seconds 601-1200 the positive interaction context. 
Table 2

Change Point Results for Correlation (r), Instantaneous Derivative Matching (ISM), and Signal Matching (SM)

\begin{tabular}{|c|c|c|c|c|c|}
\hline & \multirow[t]{3}{*}{ Location } & \multirow{3}{*}{$\begin{array}{l}\text { Number of couples } \\
\text { with change points }\end{array}$} & \multicolumn{3}{|c|}{ Number of change points } \\
\hline & & & & \multicolumn{2}{|c|}{ Direction of change } \\
\hline & & & & + & - \\
\hline \multirow{4}{*}{$r$} & Total & 2 & 3 & 0 & 3 \\
\hline & Transition & 0 & 0 & 0 & 0 \\
\hline & Negative & 2 & 2 & 0 & 2 \\
\hline & Positive & 1 & 1 & 0 & 1 \\
\hline \multirow{4}{*}{ IDM } & Total & 33 & 49 & 17 & 32 \\
\hline & Transition & 7 & 7 & 6 & 1 \\
\hline & Negative & 19 & 21 & 5 & 16 \\
\hline & Positive & 19 & 21 & 6 & 15 \\
\hline \multirow{4}{*}{ SM } & Total & 47 & 82 & 38 & 44 \\
\hline & Transition & 3 & 3 & 2 & 1 \\
\hline & Negative & 27 & 33 & 11 & 22 \\
\hline & Positive & 34 & 46 & 25 & 21 \\
\hline
\end{tabular}

Note: Transition denotes the transition period from the negative to positive interaction context (i.e., 51 second period around the transition at 600 seconds). 
Running Head: CHANGE POINT DETECTION IN INTERPERSONAL DYNAMICS

\section{Figure 1}

Influence of Changes in Shape, Scatter, and/or Level on the Similarity Measures

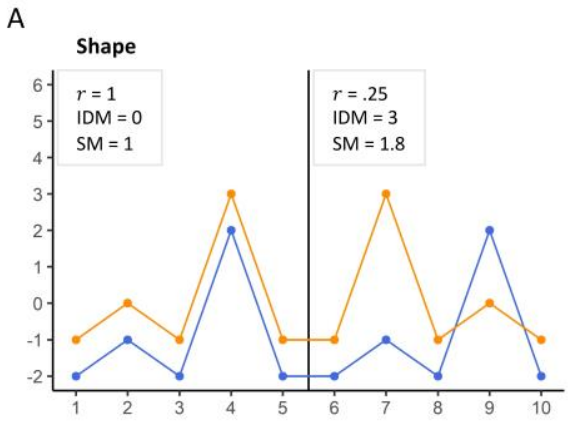

E

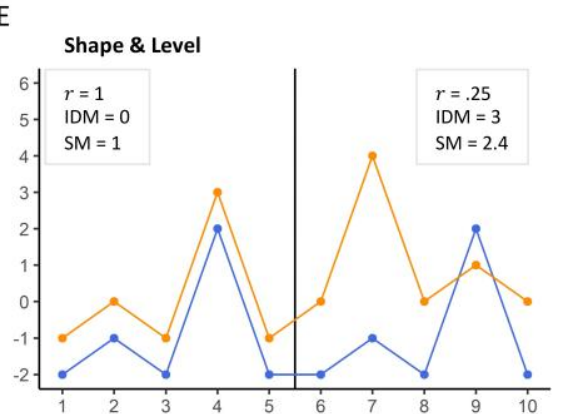

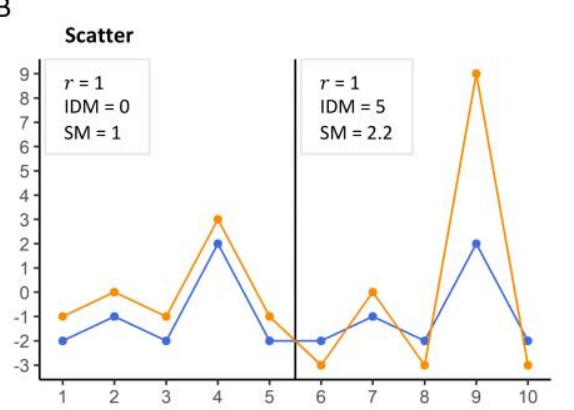

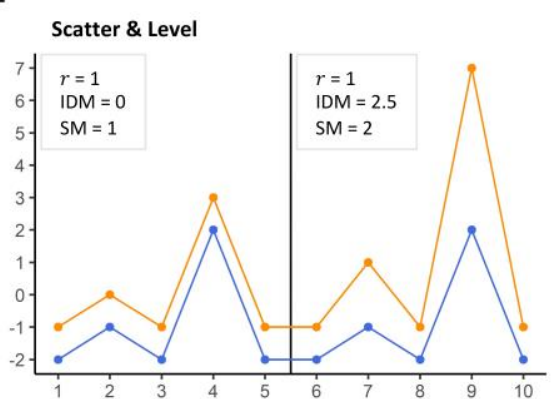

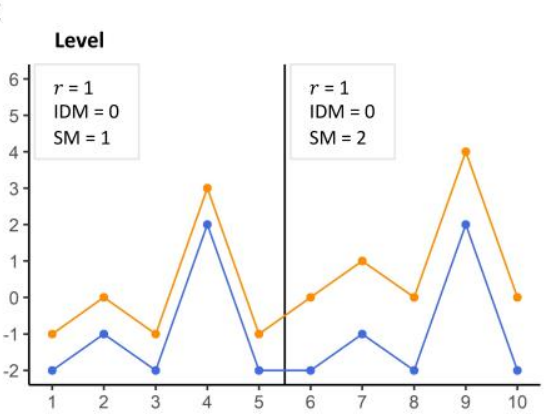

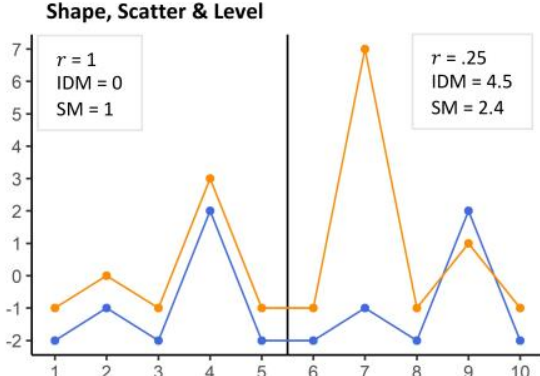

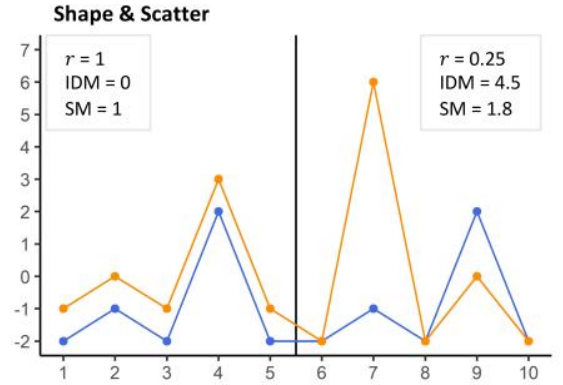

Note: $r$, IDM, and SM denote the correlation, instantaneous derivative matching, and signal matching. 


\section{Figure 2}

Time Series Data of the Example Couple

A
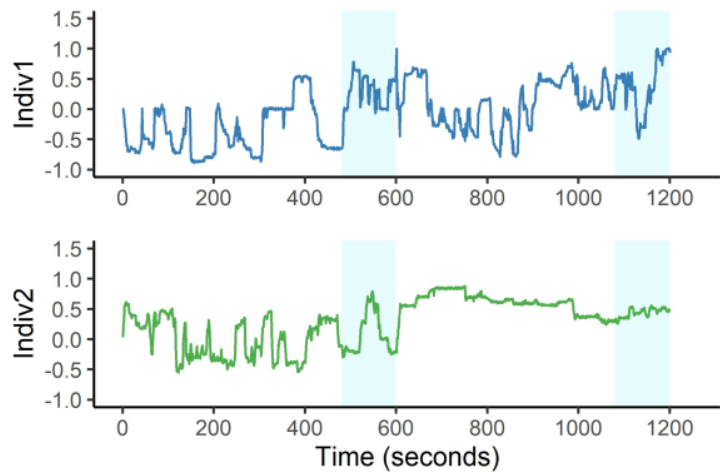

C
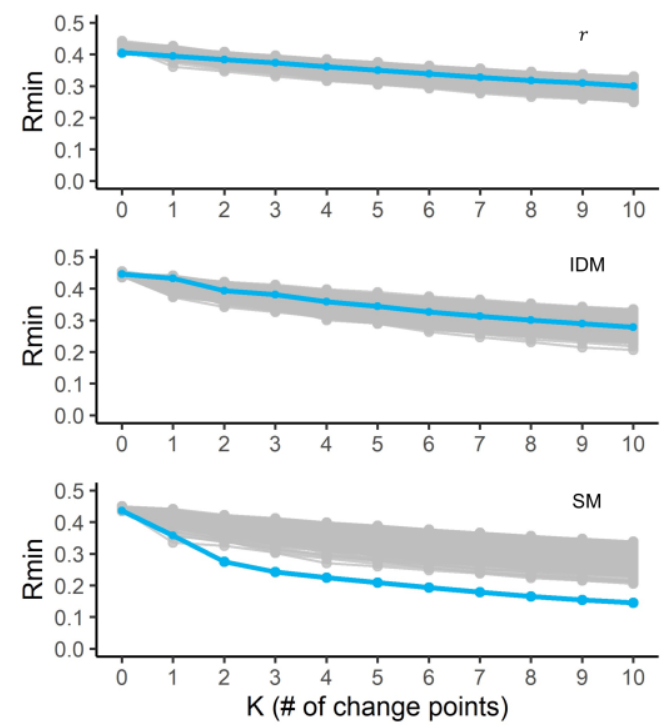
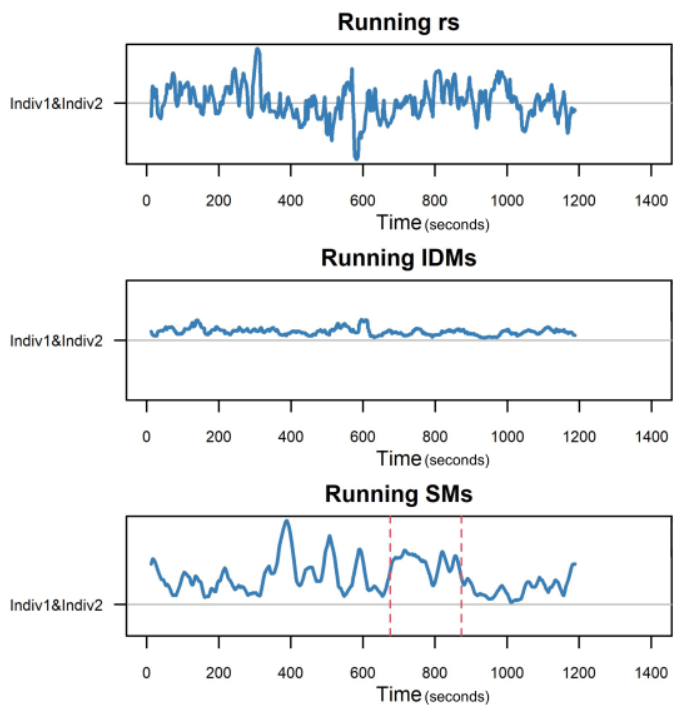

D

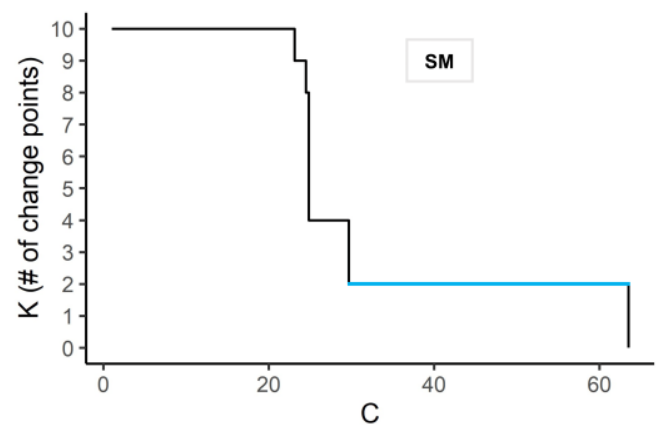

Note: (a) The observed scores of both partners, where Indiv1 is the male individual and

Indiv2 the female individual. Seconds 1-600 correspond to the negative interaction context and seconds 601-1200 to the positive one. The blue shadings indicate the periods after the knock on the door. (b) The running values for correlation $(r)$, instantaneous derivative matching (IDM), and signal matching (SM) where the red vertical lines indicate the detected change points at 676 and 873 seconds. (c) $\hat{R}_{\text {min,K }}$ values for each running statistic, plotted against the number of change points. The blue and grey lines indicate the values for the observed and permuted data, respectively. (d) Results of the grid search for the running SMs. 


\section{Figure 3}

Detected Correlation (r), Instantaneous Derivative Matching (IDM), and Signal Matching (SM) Change Points Per Couple, Each Represented by a Different Shape.

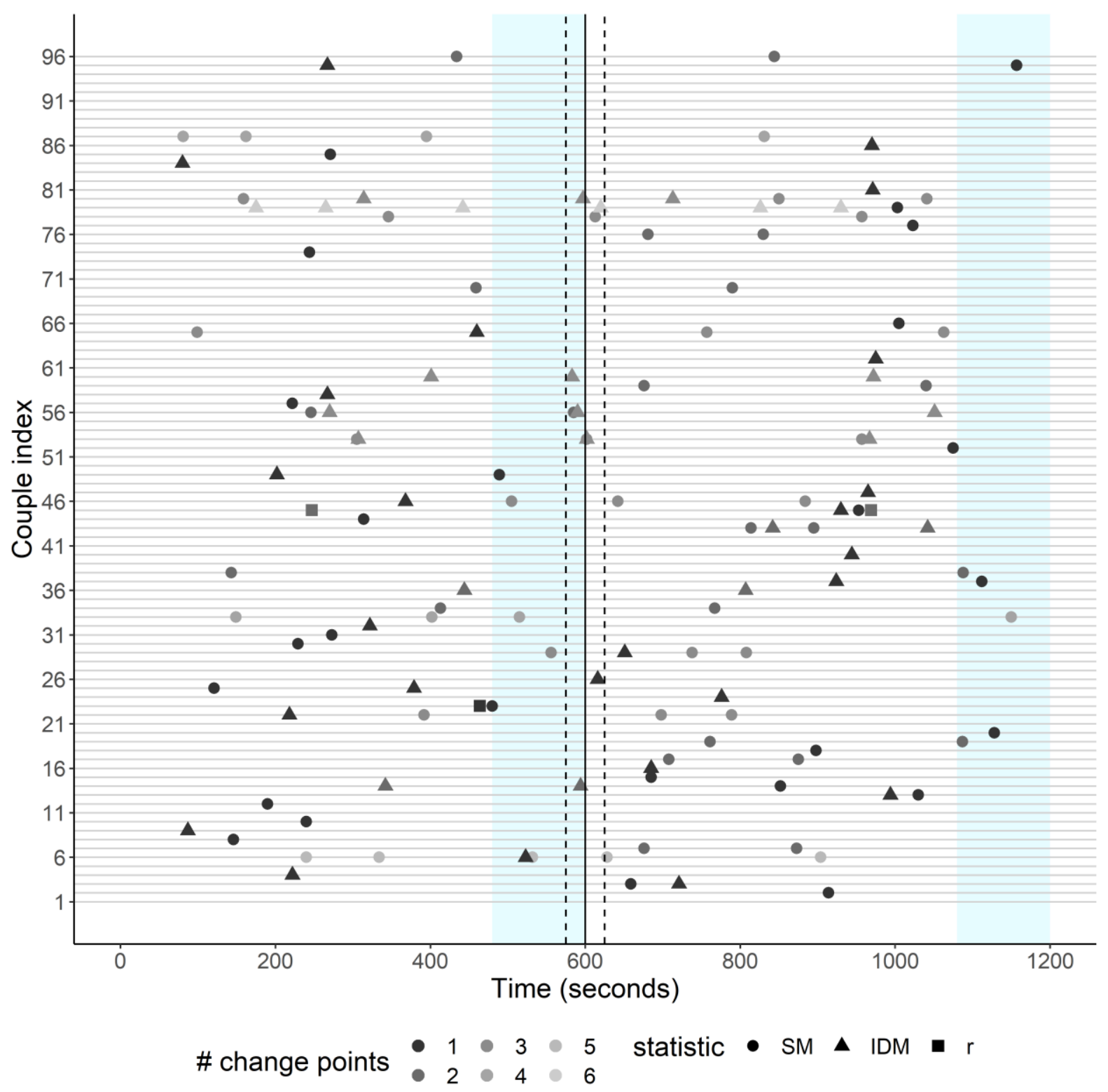

Note: The colors indicate the number of change points found per couple and per statistic. The vertical line at 600 seconds indicates the transition from the negative to positive interaction context. The dashed vertical lines denote the 51 second period around the transition. The blue shadings indicate the periods after the knock on the door. 


\section{Figure 4}

The Observed Scores of Our Example Couple
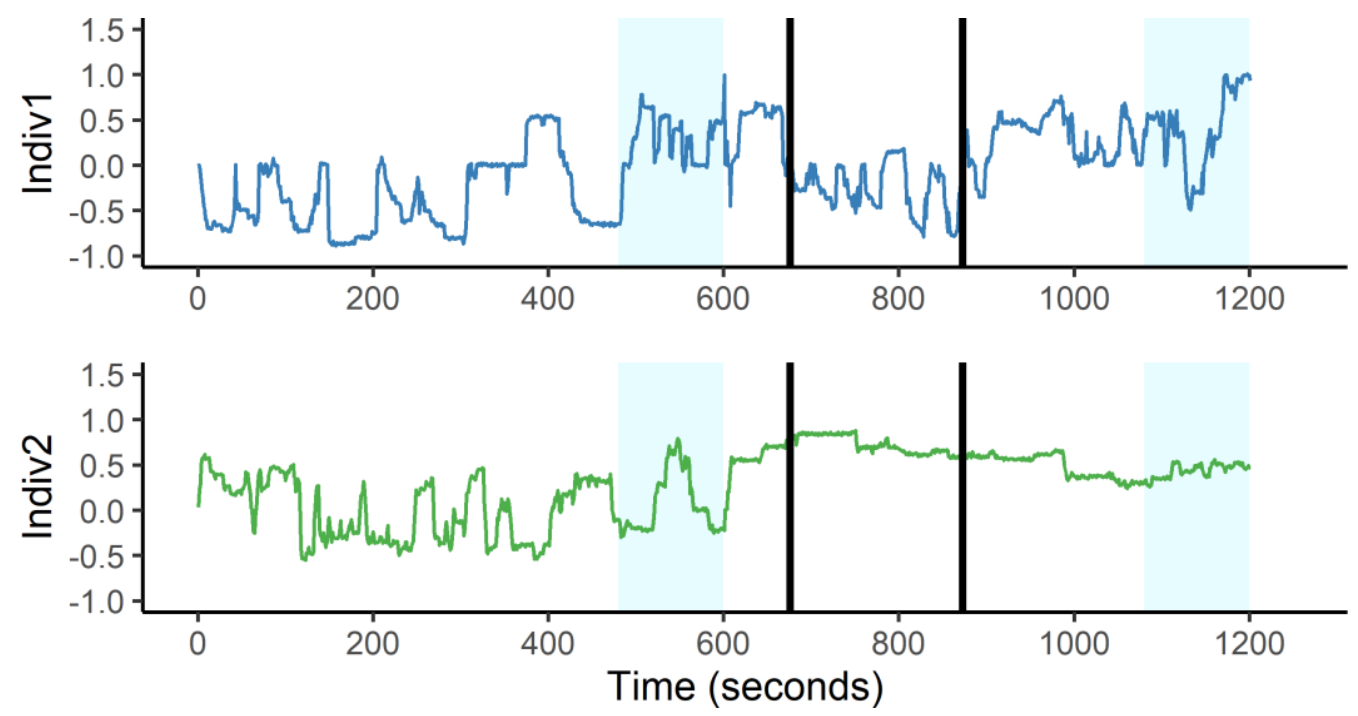

Note: Indiv1 is the male individual and Indiv2 the female individual. The blue shadings indicate the periods after the knock on the door. The two solid vertical lines indicate the detected signal matching change points at 676 and 873 seconds. 